\title{
CUIDADOS DE ENFERMAGEM AO RECÉM-NASCIDO COM CATETER CENTRAL DE INSERÇÃO PERIFÉRICA
}

\section{ARTIGO DE REVISÃO}

BATISTA, Marcos Antonio Silva ${ }^{1}$

OLIVEIRA, Carla Karolina de Almeida ${ }^{2}$

MENDES, Márcio Martins ${ }^{3}$

GONÇALVES, Rosane Cristina Mendes ${ }^{4}$

BATISTA, Marcos Antonio Silva. Et al. Cuidados de enfermagem ao recém-nascido com cateter central de inserção periférica. Revista Científica Multidisciplinar Núcleo do Conhecimento. Ano 05, Ed. 11, Vol. 20, pp. 29-42. Novembro de 2020. ISSN: 24480959, Link de acesso: https://www.nucleodoconhecimento.com.br/saude/insercaoperiferica

\section{RESUMO}

$\mathrm{Na}$ Unidade de Terapia Intensiva Neonatal (UTIN) o uso do Cateter Central de Inserção Periférica (PICC) apresenta-se bastante difundido por inúmeras vantagens e benefícios para os pacientes, sendo a instalação do PICC uma das intervenções assistenciais realizadas por enfermeiros. Este trabalho tem como objetivo avaliar os cuidados do enfermeiro ao recém-nascido na UTIN quanto à inserção e manutenção do PICC. Trata-se de uma revisão de literatura, foi realizada nas bases de dados LILACS, BVS, MEDLINE e na Base de Dados de Enfermagem-BDENF. É de responsabilidade do enfermeiro, privativamente, a assistência e cuidados de

\footnotetext{
${ }^{1}$ Especialista em Enfermagem do Trabalho pela Faculdade Gianna Bereta.

${ }^{2}$ Especialista em Enfermagem Obstétrica pelo Instituto Superior Santa Cecília - IESC.

${ }^{3}$ Graduado em Enfermagem pelo Centro Educacional Anhanguera de Santo André.

${ }^{4}$ Especialista em Saúde Pública pela Universidade Federal do Tocantins - UFT.
} 
enfermagem de maior complexidade técnica, que demandam estudos e conhecimentos de base científica, além de habilidade para tomar providência imediata, portanto, a técnica de implantação e manuseio do PICC é de incumbência desse profissional. Conclui-se que o enfermeiro tem um papel importante nos cuidados para minimizar as causas de remoção não eletiva do PICC, sendo relevante destacar a capacitação e aprimoramento da habilidade do profissional quanto à inserção, manutenção, retirada e observação desse dispositivo.

Palavras-chave: Neonato, enfermagem, Unidade de Terapia Intensiva, cateter.

\section{INTRODUÇÃO}

O trabalho do profissional de enfermagem na Unidade de Terapia Intensiva Neonatal (UTIN) é de grande responsabilidade, pois este profissional presta cuidados diretos às peculiaridades do recém-nascido $(\mathrm{RN})$. A crescente prática de procedimentos de alto risco e a intolerância a erros de medicação faz com que tal responsabilidade seja redobrada. Além disso, é o enfermeiro quem garante a assistência à família e o cuidado ao desenvolvimento físico, psíquico e social do recém-nascido, e ainda é este profissional que está lado a lado ao médico na definição de condutas de tratamento (MONTANHOLI; MERIGHI; JESUS, 2011).

Várias são as intervenções que se realizam nos recém-nascidos na UTIN, e as mais frequentes realizadas por estes profissionais é a instalação do PICC (Cateter Central de Inserção Periférica), caracterizado por ser um dispositivo vascular de inserção periférica com localização central, possuindo um ou mais lúmen. Este cateter vem sendo utilizado amplamente em pacientes em terapia intravenosa, uma vez que está associado ao menor risco de complicações mecânicas e infecciosas. Dependendo do quadro clínico e da gravidade do recém-nascido, determina-se o tipo de terapia intravenosa que será administrada, ou seja, qual o cateter apropriado ao tratamento (LOUREÇO e OHARA, 2010).

O uso do PICC apresenta-se bastante difundido por inúmeras vantagens e benefícios, e o enfermeiro é o profissional com a qualificação, capacitação, habilidades e 
conhecimentos necessários à indicação, inserção e manutenção do PICC, e isso gera uma atenção legitimada possibilitando a autonomia profissional (PAES; LEITE; MELLO, 2011).

Segundo Gomes e Nascimento (2013), o enfermeiro é legalmente amparado para inserir e manusear o PICC, sendo ele habilitado pelo título de especialista e ainda sido submetido a capacitações. São privativos deste profissional os cuidados de maior complexidade e que requeiram conhecimentos de base científica para tomada de decisões urgentes. É o enfermeiro que faz a escolha do acesso venoso a ser utilizado, faz a manutenção para que não haja perigo à criança e monitoramento para uma terapêutica segura para afastar e/ou diminuir as intercorrências.

Podem-se citar ainda procedimentos que o enfermeiro deve estar atento nos quais são: administração de soluções ou fármacos, preparar o $\mathrm{RN}$ para o procedimento, manutenção do cateter venoso periférico, cuidados com a frequência de troca do cateter, dispositivo de infusão soluções e curativos (SANTOS e MARTINS, 2014).

Dessa forma, o objeto de estudo deste trabalho é avaliar os cuidados do enfermeiro ao recém-nascido na Unidade de Terapia Intensiva Neonatal quanto a inserção e manutenção do Cateter Central de Inserção Periférica.

\section{REFERENCIAL TEÓRICO}

O profissional enfermeiro tem competência técnica e legal para indicação, introdução e manuseio da PICC, sendo este amparado pela Resolução COFEN n 258 de 2001. No entanto, segundo a resolução, o profissional deve se submeter a uma qualificação ou capacitação profissional, buscando sua autonomia frente ao procedimento e para demonstrar competência, aptidão e responsabilidade (LUCA, 2013), e, além disso, o enfermeiro deve estar apto para prevenção de complicações (MOTTA, 2011).

É importante salientar, que o juízo das condições clínicas do RN para iniciar a introdução do PICC é de fundamental importância, sendo que, tem-se que avaliar perfusão periférica, e essa estar satisfatória, normotermia, hidratação e nível de 
oxigenação acima de $90 \%$. É cabível também que este procedimento não seja realizado em causas de urgência (LUCA, 2013).

Sabendo que o PICC não é um procedimento de emergência e que às vezes a equipe médica faz a indicação sem o prévio conhecimento das técnicas de indicação, o enfermeiro como sujeito habilitado e com conhecimentos para tal procedimento tem que mostrar sua autonomia e entrar em consenso com os médicos, demonstrando assim o porquê da não indicação no momento e aguardar para que se possa realizar a colocação do cateter, ou seja, estabilizar o quadro do RN (LUCA, 2013).

No entanto, ainda de acordo com Luca (2013), para que sua autonomia frente ao PICC seja respeitada e reconhecida dentre as demais categorias, tal profissional deve demonstrar seu conhecimento à cerca do procedimento, este seguido de suas habilidades e responsabilidades. É de fundamental importância que o profissional enfermeiro se mostre bem seguro de suas ações e que sempre deva ser incitado a sua autonomia para que o profissional possa saber se eximir de prováveis conflitos que possam aparecer.

No que diz respeito à escolha do local de inserção do cateter existem várias opções onde os critérios serão determinados pelo enfermeiro. Baggio; Bazzi; Bilibio (2010), fala que, as veias de primeira escolha são a basílica e a cefálica, seguidas da veia auricular, jugular, axilar, mediana cubital, e safena interna.

Em relação a pele do local da punção, esta deve estar íntegra, sem apresentar sinais flogísticos e sem quaisquer tipo de alterações anatômicas (LUZ, 2010).

As veias basílicas e cefálicas são destacadas como de primeira escolha, por ser um vaso de grande dimensão, ou seja, de grosso calibre, menos curvada e com menor número de válvulas (BRASIL, 2008).

Segundo Malagutti e Roehrs (2012), para a seleção da veia para punção existem várias características que indicam que o acesso é apto para a introdução do PICC, a veia deve ser macia, sem desvios, ou seja, retilínea, apresente um calibre apropriado, a pele deve estar íntegra e a veia dever ter uma boa visualização. 
A técnica de inserção do cateter consta com um procedimento totalmente estéril e algumas peculiaridades a respeito de como este cateter chegar ao local central desejado, onde este é feito por meio da mensuração com uma fita métrica. É de fundamental importância, logo no início do procedimento, acionar o serviço de radiologia, pois, ao término da inserção, o paciente será encaminhado para radiografia para certificação do local da ponta do cateter (LUZ, 2010).

Após a indicação e inserção os enfermeiros juntamente com a equipe de enfermagem devem ficar atentos com os cuidados desse cateter, ou seja, a sua manutenção. Sendo inegáveis os benefícios da terapia infusional citada, vários são os riscos em relação ao manuseio do cateter, são eles principalmente os riscos de complicações (LUCA, 2013). As complicações podem levar a remoção antecipada do dispositivo e as principais causas dessa remoção estão relacionadas obstrução, infiltração, suspeita de contaminação, tração, ruptura e retirada acidental (BAGGIO; BAZZI; BILIBIO, 2010). Chamando a atenção para o potencial risco de infecção e o profissional deve estar em alerta para isso, buscando sempre a forma mais correta de manipulação para diminuir tais riscos e estando preocupado com o bem estar do paciente (LUCA, 2013).

O risco de infecção está presente sempre que a manipulação do dispositivo for inadequada, o curativo realizado de forma correta propicia um ambiente de proteção, é realizado em procedimento totalmente estéril para afastar micro-organismos do local inserção do cateter, onde este é um local propício à infecção e complicações resultando em remoção do dispositivo antes do tempo indicado (LUZ, 2010).

É o enfermeiro o personagem essencial para a prevenção das complicações, sabendo que ele é que se responsabiliza pela reabilitação do paciente e êxito no tratamento (JANTSCH et al., 2014). 


\section{METODOLOGIA}

O estudo trata-se um uma revisão integrativa de literatura, para a sua construção optou-se por seguir as etapas propostas por Sousa; Silva; Carvalho (2010), que possibilitou uma maior clareza dos passos utilizados para a realização deste estudo.

A seis etapas foram: identificação do tema e seleção da questão norteadora; estabelecimento de critérios para inclusão e exclusão de estudos e busca na literatura pertinente; definição das informações a serem extraídas dos estudos selecionados; avaliação crítica dos estudos incluídos na revisão literária; interpretação dos resultados; apresentação da revisão/síntese do conhecimento.

A coleta de dados foi realizada de julho a agosto de 2019 por meio da consulta direta a internet no endereço eletrônico do banco de dados da Literatura Latino-americana e do Caribe em Ciências da Saúde (LILACS) e Biblioteca Virtual em Saúde (BVS), onde foram acessadas as bases de dados MEDLINE (Medical Literature Analysis and Retrieval System Online) e da Base de Dados de Enfermagem-BDENF.

Para realizar as buscas nas bases de dados acima citas, foram utilizados os termos em saúde obtidos junto aos Descritores em Ciências da Saúde (DeCS) Os descritores "Nonato", "Enfermagem", “Unidade de Terapia Intensiva" e "Cateter", foram cruzados através do operador booleano AND, sendo ele uma combinação aditiva.

A seleção dos dados foi baseada nos seguintes critérios de inclusão: artigos dos últimos oito anos (2011-2019), em português disponível na íntegra, que abordavam a temática em estudo independentemente do método de pesquisa utilizados.

Como critério de exclusão, optou-se por não utilizar estudos que não fossem artigos, que estivessem em língua estrangeira, não estivessem disponíveis na íntegra, não fossem dos últimos oito anos e que não se relacionasse ao objeto do estudo ou que não fornecessem informações suficientes sobre a temática abordada.

A análise das publicações iniciou em julho e finalizou em agosto de 2019, por meio de levantamento bibliográfico, tendo como foco o tema: Cateter central de inserção 
periférica em recém-nascidos na UTI neonatal. Após a análise temática dos títulos e resumos, os artigos obtidos foram caracterizados para organizar os resultados. As características dos artigos incluídos foram organizadas de forma clara e apresentados em uma tabela para permitir ao leitor uma avaliação clara sobre a natureza dos mesmos.

A presente revisão integrativa de literatura assegura todos os aspectos éticos, garantindo a autoria dos artigos pesquisados, sendo os autores citados tanto no corpo do texto como nas respectivas referências deste trabalho, obedecendo às normas da Associação Brasileira de Normas Técnicas (ABNT).

\section{RESULTADOS E DISCUSSÕES}

Para melhor apresentar o perfil dos artigos incluídos pesquisa foi realizada a caracterização dos mesmos, a qual utilizou-se de procedimentos que envolveram: o periódico, número de publicações por periódico e ano de publicação dos estudos (Tabela1).

De acordo com a tabela 1, observa-se que a Revista Brasileira de Enfermagem e a Revista da Escola de Enfermagem da USP foram os únicos periódicos que apresentaram duas publicações, correspondendo juntos por aproximadamente 33\% dos artigos selecionados para compor a discussão deste estudo. Os demais apresentaram em sua composição apenas um estudo (aproximadamente $8 \%$ ) e juntos corresponderam por aproximadamente $67 \%$ das publicações totais.

Foi possível identificar que dos estudos incluídos nesta revisão o ano de 2013 foi o ano em que houve o maior número de estudos publicados, com um total de quatro publicações, seguido dos anos de 2012 com três publicações e 2011 com duas. Só obtivemos um artigo em cada um dos demais anos (2014, 2015 e 2018).

Apesar de os pesquisadores utilizarem o ano o período temporal de 2011 a 2019 como um filtro para inclusão, não houve pesquisas nos anos de 2016, 2017 e 2019 que atendesse todos os critérios estabelecidos para compor este estudo. 
Tabela 1 - Distribuição dos artigos científicos segundo o ano de publicação, autor, periódico e títulos dos artigos

\begin{tabular}{|c|c|c|c|}
\hline ANO & AUTOR & PERIÓDICO & TÍTULO \\
\hline 2011 & BARBOSA & $\begin{array}{l}\text { J. res.: fundam. } \\
\text { care. online }\end{array}$ & $\begin{array}{l}\text { The importance of nurses in the } \\
\text { handling of the picc in the neonatal } \\
\text { intensive care unit }\end{array}$ \\
\hline 2011 & DÓREA et al., & $\begin{array}{l}\text { Rev. bras. } \\
\text { enferm, }\end{array}$ & $\begin{array}{l}\text { Práticas de manejo do cateter } \\
\text { central de inserção periférica em } \\
\text { uma unidade neonatal }\end{array}$ \\
\hline 2012 & BELO et al., & $\begin{array}{l}\text { Rev. bras. } \\
\text { enferm }\end{array}$ & $\begin{array}{l}\text { Conhecimento de enfermeiros de } \\
\text { neonatologia acerca do cateter } \\
\text { venoso central de inserção } \\
\text { periférica }\end{array}$ \\
\hline 2012 & COSTA; PAES & Esc. Anna Ner & $\begin{array}{l}\text { Aplicabilidade dos diagnósticos de } \\
\text { enfermagem como subsídios para } \\
\text { indicação do cateter central de } \\
\text { inserção periférica }\end{array}$ \\
\hline 2012 & JOHANN et al & $\begin{array}{l}\text { Rev. esc. enferm. } \\
\text { USP }\end{array}$ & $\begin{array}{l}\text { Cuidados com cateter central de } \\
\text { inserção periférica no neonato: } \\
\text { revisão integrativa da literatura }\end{array}$ \\
\hline 2013 & DUARTE et al. & $\begin{array}{l}\text { Rev. esc. enferm. } \\
\text { USP }\end{array}$ & $\begin{array}{l}\text { Fatores associados à infecção pelo } \\
\text { uso do cateter central de inserção } \\
\text { periférica em Unidade de Terapia } \\
\text { Intensiva Neonatal }\end{array}$ \\
\hline 2013 & $\begin{array}{l}\text { GOMES; } \\
\text { NASCIMENTO }\end{array}$ & $\begin{array}{l}\text { Revista Escola } \\
\text { Enfermagem }\end{array}$ & $\begin{array}{l}\text { O processo do cateterismo venoso } \\
\text { central em Unidade de Terapia } \\
\text { Intensiva Neonatal e Pediátrica. }\end{array}$ \\
\hline 2013 & $\begin{array}{l}\text { MOURA; } \\
\text { CONTIM; } \\
\text { AMARAL; }\end{array}$ & $\begin{array}{l}\text { Rev. enferm. } \\
\text { atenção, saúde }\end{array}$ & $\begin{array}{l}\text { Cateter central de inserção } \\
\text { periférica: revisão bibliométrica. }\end{array}$ \\
\hline
\end{tabular}




\begin{tabular}{|c|c|c|c|}
\hline 2013 & CABRAL et al., & $\begin{array}{l}\text { Revista } \\
\text { Eletrônica de } \\
\text { Enfermagem }\end{array}$ & $\begin{array}{l}\text { Análise do uso de cateter central de } \\
\text { inserção periférica em Unidade de } \\
\text { Cuidado Intensivo Neonatal. }\end{array}$ \\
\hline 2014. & $\begin{array}{l}\text { COSTA;SILVA; } \\
\text { KIMURA }\end{array}$ & Online braz j nurs & $\begin{array}{l}\text { Intravenous therapy and non- } \\
\text { elective removal of epicutaneous } \\
\text { catheters: a cohort study of } \\
\text { neonates }\end{array}$ \\
\hline 2015 & $\begin{array}{l}\text { VERA; SOUSA; } \\
\text { MESQUITA }\end{array}$ & $\begin{array}{l}\text { Rev Ciência } \\
\text { \&amp; Saberes } \\
\text { Facema }\end{array}$ & $\begin{array}{l}\text { A atuação do enfermeiro na prática } \\
\text { deinserção e manutenção do PICC: } \\
\text { Uma revisão integrativa de } \\
\text { literatura. }\end{array}$ \\
\hline 2018 & LUI et al., & RECOM & $\begin{array}{l}\text { Cuidados e limitações no manejo do } \\
\text { cateter central de inserção } \\
\text { periférica em neonatalogia }\end{array}$ \\
\hline
\end{tabular}

FONTE: Dados coletados pelos autores.

\subsection{CUIDADOS DO ENFERMEIRO DESDE A INSERÇÃO ATÉ A RETIRADA DO PICC NO RN EM UMA UTIN}

Nesta categoria foram selecionadas nove produções científicas para elucidar a importância do profissional enfermeiro na manipulação do PICC nos RN's.

De acordo com Gomes e Nascimento et al. (2013), é de responsabilidade do enfermeiro, privativamente, a assistência e cuidados de enfermagem de maior complexidade técnica e que demandam estudos e conhecimentos de base científica e habilidade de tomar providencias imediatas, a técnica de implantação e manuseio do PICC é de incumbência desse profissional. Assim sendo, o enfermeiro deve eleger apropriadamente o acesso venoso a ser empregado, e optar por precauções que favoreçam a preservação desse acesso sem perigos, riscos ou danos à criança, assegurando, assim, a execução fiel da terapêutica intravenosa e colaborando para a diminuição do estresse. Moura, Contim e Amaral (2013) concordam e acrescentam 
que médicos devidamente treinados e capacitados também podem realizar o procedimento.

Segundo Barbosa (2011), a terapia intravenosa é um tipo de procedimento que é de total responsabilidade da enfermagem, em especial do enfermeiro, onde este exerce um papel primordial no cuidado referente aos cateteres venoso central de inserção periférica (PICC) que vem sendo aplicado nas terapias da maioria das unidades de Terapia Intensiva Neonatal (UTIN) como aliado no cuidado concedido aos recémnascidos ( $R N)$, contudo há a necessidade de treinamento, experiência e prática da equipe Neonatal quanto aos cuidados com a inserção e manutenção do PICC para assegurar uma melhor assistência a estes recém-nascidos, e também estar utilizando dessa sofisticação tecnológica adequadamente. Belo et al., (2012) e Duarte et al., (2013), concordam com o citado anteriormente e completam que com a presença e o uso de protocolos na instituição para direcionar a técnica dos profissionais de enfermagem na utilização desse cateter propõe normatizar condutas e dar uma melhoria na qualidade da assistência, o que faz-se substancial para o sucesso do manuseio com o PICC.

Belo et al., (2012), diz que no tocante à indicação do PICC, quando é inquestionável manter um acesso venoso profundo por tempo demorado (acima de uma semana), infusão de soluções hiperosmolares, vesicantes e irritantes. Vera, Sousa e Araújo (2015) adicionam ainda que também é indicado para administração de nutrição parenteral com concentração de dextrose maior que 10\%; infusão de medicamentos, vasoativos, de soluções ou com pH não fisiológico, a exemplo de alguns antibióticos e de quimioterápicos antineoplásicos; administração de hemoderivados, medida de pressão venosa central e coleta de sangue

A seleção da veia para fazer a punção é de extrema importância para o êxito da inserção do cateter. As veias cefálica e a basílica são as mais eleitas para o processo, sendo a basílica, a mais recomendada em razão de seu maior calibre, menor número de válvulas e a de mais fácil manipulação no procedimento de troca de curativo (DÓREA et al., 2011; LUl et al., 2018). 
Costa et al. (2012), explica melhor os motivos para a veia de acesso mais recomendada para inserção desse dispositivo vascular em neonatos ser a basílica, de acordo com ele é a que demanda um trajeto menor para o curso do cateter também apresenta maior diâmetro luminal na região cubital. Sua rota segue a face interna do braço e possui entre 4 e 8 válvulas venosas. A veia cefálica, no entanto, apresenta um maior percurso até a veia cava podendo assim potencializar o perigo para desenvolvimento de flebite e mau instalação da ponta do cateter, também está localizada nos membros superiores, tendo esta um diâmetro luminal inferior comparada a basílica, possui entre 6 e 8 válvulas venosas e seu percurso segue a face externa do braço contornando o ombro.

Johann et al., (2012) discutiu essa mesma temática dizendo que no que se refere ao sítio de introdução do cateter, não há consenso na literatura sobre o melhor localização para tal população. Ele relata que existem estudos que apontam que a utilização do PICC em membros inferiores, quando for possível, deve ser levada em conta para infusão prolongada de Nutrição Parenteral Total. Porém, estudos também mostram que as veias femoral e subclávia têm maior incidência para eventos venosos tromboembólicos, sendo mais aconselhável sua inserção em veia jugular ou braquial.

Ao se tratar da manutenção, está apontada a remoção no momento em que o cliente não precise mais do dispositivo, bem como observação diária do local de inserção, a fim de supervisionar sinais flogísticos a fim de evitar complicações. Não existe advertência para o uso de antibióticos profiláticos sistêmicos antes da implantação ou mesmo durante a estadia do cateter. Contudo, a aplicação tópica de pomadas antibióticas não é indicada pelo potencial de resistência microbiana e infecções fúngicas (VERA; SOUSA; MESQUITA, 2015; LUI et al., 2018).

Barbosa (2011) afirma que, para que seja alcançado o sucesso da utilização do PICC, é necessário que os profissionais de enfermagem busquem o conhecimento técnico e cientifico por meio de treinamentos e habilitações, para evitar complicações e saber intervir diante de problemas que possam surgir ou que já se encontram instalados. 
Cabral et al., (2013) ainda considera precaver contra a infecção do cateter um fator prioridade para profissionais de saúde, tendo que haver uma atenção sistemática e rigorosa quanto à higienização das mãos, os manejos assépticos para com o cateter e bastante cautela e cuidado com o sítio de inserção do dispositivo. Há uma redução da quantidade de infecções quando existem precauções particulares com o cateter que são impostas por meio do emprego de protocolos e profissionais bem treinados. Vera, Sousa e Mesquita (2015) completa dizendo que também considera que pode vir do profissional de saúde esses agentes infecciosos, e salienta ainda sobre as precauções que devem ser usadas por estes que são os equipamentos de proteção individual: máscara, gorro, avental estéril, luvas e campos estéreis.

Segundo Belo et al., (2012) a observação das complicações relativas à infecção é de total responsabilidade do enfermeiro, pois a vigilância dos sinais como hipertermia, rubor e secreção no óstio da inserção devem ser executadas cotidianamente. Constatando que os profissionais treinados e familiarizados com a técnica do PICC são de real importância para que as condutas sejam aplicadas no caso das complicações precocemente. Costa, Silva e Kimura (2014), complementa dizendo que a infecção na corrente sanguínea relacionada ao uso de PICC é um risco pertinente ao emprego de qualquer dispositivo de acesso vascular seja do mais simples acesso ao mais complicado. No que se trata dos fatores de risco, alguns incluem a prematuridade, no caso neonatos com peso ao nascer inferior a $1.000 \mathrm{~g}$ em razão de deficiências em seu sistema imunológico e grande número de procedimentos invasivos a que estão sujeitos durante a internação na UTIN. Outros fatores incluem: inexperiência da equipe de enfermagem no cuidado diário do cateter, excesso na manipulações do cateter, contaminação do canhão do cateter e o longo tempo de permanência do mesmo.

Dórea et al. (2011), afirmaram que várias são as complicações encontradas mediante o uso do PICC, e relataram em suas pesquisas que as mais encontradas foram: a obstrução intralúmen do cateter que pode vir a acontecer por trombos sanguinolentos ou pelo desenvolvimento de fibrina em consequência da existência de sangue no cateter logo depois de uma lavagem inadequada do cateter ou fluxo retrógrado; a 
obstrução, por outro lado, pode ser de etiologia não trombótica, ocasionada por minerais precipitados, provenientes de soluções infundidas ou medicamentos incompatíveis e a ruptura do cateter, uma outra complicação que está diretamente ligada ao mau manuseio do cateter e à infusão com grande pressão intralúmen. A contaminação do cateter pode acontecer através da entrada direta dos microorganismos presentes na pele e no sítio de inserção do cateter, por manuseio inapropriado de soluções parenterais e conexões do cateter ou contaminação endógena.

Vera, Sousa e Mesquita (2015) concorda com o autor Costa, Silva e Kimura (2014) e ainda acrescenta que a flebite, a infiltração e o extravasamento são as complicações mais frequentemente relacionadas ao uso de cateteres venosos periféricos (CVP). Também cita que alguns fatores que podem contribuir para a ocorrência dessas complicações são o tipo de cateter utilizado, o preparo do local da punção, o tipo de infusão, a técnica de inserção e as características intrínsecas do paciente.

Belo et al. (2012), confere com os demais autores em relação as principais complicações relacionadas à pós-inserção do cateter, que são elas: obstrução e migração do cateter, ruptura desse com potencial risco de embolia e trombose, infecção sistêmica e ainda acrescenta flebite, celulite.

No estudo de Cabral et al. (2013), ele apresenta que resultados de culturas das pontas dos cateteres evidenciaram que o agente predominante foi o Staphylococcus coagulasse negativo. Este micro-organismo pertence à microbiota normal da pele, $\mathrm{e}$ se torna muitas vezes um agente oportunista, no caso de recém-nascidos principalmente com o uso de cateter venoso central. Além da origem endógena, da pele e mucosa do neonato, pode ter origem exógena, ou seja, no meio do ambiente hospitalar ou nas mãos dos profissionais de saúde esse micro-organismo é bastante comum. 


\section{CONSIDERAÇÕES FINAIS}

O enfermeiro tem um papel importante nos cuidados para minimizar as causas de remoção não eletiva do PICC, sendo relevante destacar a capacitação e aprimoramento da habilidade do profissional quanto à inserção, manutenção, retirada e observação desse dispositivo. Acrescenta-se ainda a importância da técnica correta para antissepsia na inserção e manutenção do mesmo, uma vez que a pele do neonato é delicada e requer um maior cuidado por parte da equipe de enfermagem.

Levando-se em conta o que foi observado, conclui-se, portanto, que, o enfermeiro devidamente capacitado na técnica de inserção do cateter central periférico é de grande importância, pois os estudos mostraram que a técnica sendo bem empregada diminui significativamente os riscos de infecção e complicações advindas do procedimento, porém, quando não acertada, podem trazer algumas complicações aqui já expostas.

\section{REFERÊNCIAS}

BAGGIO, M. A; BAZZI, F. C. S; BILIBIO, C. A. C. Cateter central de inserção periférica: descrição da utilização em UTI Neonatal e Pediátrica. Rev. Gaúcha Enferm. (Online), Porto Alegre, v. 31, n. 1, p. 70-76, mar. 2010.

BARBOSA, J. P. The importance of nurses in the handling of the picc in the neonatal intensive care unit. Revista de Pesquisa: Cuidado é fundamental online, v. 3, n. 2, p. 24, 2011.

BELO, M. P. M. et al. Conhecimento de enfermeiros de neonatologia acerca do cateter venoso central de inserção periférica. Rev. bras. enferm, v. 65, n. 1, p. 42-48, 2012.

CABRAL, P. F. A. et al. Análise do uso de cateter central de inserção periférica em Unidade de Cuidado Intensivo Neonatal. Revista Eletrônica de Enfermagem, v. 15, n. 1, p. 96-102, 2013. 
COSTA, L. C.; PAES, G. O. Aplicabilidade dos diagnósticos de enfermagem como subsídiospara indicação do cateter central de inserção periférica. Esc. Anna Ner, Rio de Janeiro, RJ,vol.16, n. 4, p.649-656, 2012.

COSTA, P. SILVA, M. N. KIMURA, A. F. Intravenous therapy and non-elective removal of epicutaneous catheters: a cohort study of neonates . Online braz j nurs [internet]. v. 13. n. 2. p. 129-138. 2014.

DUARTE, E. D. et al. Fatores associados à infecção pelo uso do cateter central de inserção periférica em Unidade de Terapia Intensiva Neonatal. Revista da Escola de Enfermagem da USP, v. 47, n. 3, p. 547-554, 2013.

GOMES, A. V. O.;NASCIMENTO, M. A. L. O. processo do cateterismo venoso central em Unidade de Terapia Intensiva Neonatal e Pediátrica. Revista Escola Enfermagem. São Paulo, SP, v. 47, n. 4, p. 794 - 800, Ago. 2013.

JANTSCH, L. B. et al. Utilização do cateter central de inserção Periférica em neonatologia. Revista Baiana de Enfermagem. Salvador, BA. v. 28, n. 3, p. 244-251, Set./Dez. 2014.

JOHANN, D. A. et al. Cuidados com cateter central de inserção periférica no neonato: revisão integrativa da literatura. Revista da Escola de Enfermagem da USP, v. 46, n. 6, p. 1503-1511, 2012.

LOURENÇO, S. A.; OHARA, C. V. S. Conhecimento dos enfermeiros sobre a técnica de inserção do cateter central de inserção periférica em recém-nascidos. Revista Latino-Americano Enfermagem.[S.I.: s.n]. p. 49 - 56, Mar./Abr. 2010.

LUCA, H. M. O enfermeiro e a utilização do cateter central de inserção periférica em recém-nascidos: contribuições à luz da segurança e bioética. 2013. p. 104. Dissertação (Mestrado em enfermagem) - Universidade do Estado do Rio de Janeiro. RJ, 2013. 
LUI, A. M. L et al. Cuidados e limitações no manejo do cateter central de inserção periférica em neonatalogia. RECOM. v.8, p. 1918- 1925, 2018.

\section{LUZ, S. Protocolo Instalação de Cateter Venoso Central para Inserção Periférica? PICC.}

MALAGUTTI, W.; ROEHRS, H. Terapia intravenosa: atualidades. São Paulo: Mortinari, 2012.

MONTANHOLI, L. L.; MERIGHI, M. A. B.; JESUS, M. C. P. Atuação da enfermeira na unidade de terapia intensiva neonatal: entre o ideal, o real e o possível. Revista Latino-Americano Enfermagem.[S.I.: s.n].p. 01 - 08, Mar./Abr. 2011.

MOTTA, P.N. et al. Cateter central de inserção periférica: o papel da enfermagem na sua utilização em neonatologia. HU Revista. Juiz de Fora, MG, v. 37, n. 2, p. 163-168, abr./jun. 2011.

MOURA, P. F.; CONTIM, D.; AMARAL, J. B. Cateter central de inserção periférica: revisão bibliométrica. Rev. enferm. atenção, saúde, v. 2, n. 1, p. 106-115, 2013.

PAES, G.O.; LEITE, J.L.; MELO, E.C.P. Caring for the client with acute respiratory disorders: a proposed protocol for assistance to make decisions in nursing. Online Braz J Nurs.[S.I.].v. 10, n. 1, p. 1-8, 2011.

SANTOS, R.; MARTINS, M. J. L. Manutenção do PICC: o comprometimento das técnicas de enfermagem, em uma unidade de terapia intensiva neonatal. Revista Eletrônica de Enfermagem do Vale do Paraíba, Santos, SP, v. 1, n. 07, 2014.

SOUZA, M. T.; SILVA, M. D.; CARVALHO, R. Revisão integrativa: o que é e como fazer. Einstein, v. 8, n. 1, p.102-106, Set./Dez. 2010.

Enviado: Novembro, 2020.

Aprovado: Novembro, 2020. 